\title{
Recommendations for treating electronic cigarette and heated tobacco product dependence
}

\author{
Melinda Pénzes ${ }^{1}$, Renata Solimini², Francisco M. Ruiz Dominguez ${ }^{3}$, Tamás Joó4, Constantine I. Vardavas ${ }^{5}$, Panagiotis Behrakis ${ }^{5,6,7}$
}

\section{Dear Editor,}

There is strong evidence that any clear smoking cessation advice from healthcare professionals (e.g. physicians, nurses, dentists) can increase smokers' motivation to quit ${ }^{1}$. Generally, guidelines for treating tobacco smoking dependence and supporting smoking cessation urge all health professionals to provide a minimum of brief smoking cessation advice to all patients, including current tobacco consumers to quit smoking and ex-smokers to avoid relapsing ${ }^{1,2}$. E-cigarettes and HTPs are continuing to gain popularity and acceptance by consumers worldwide ${ }^{3,4}$. However, many consumers and health professionals are uncertain about the potential benefits or adverse effects of these novel products ${ }^{5,6}$. Assessment of tobacco smoking dependence in medical practice does not usually consider e-cigarette or HTP dependence. To date, guidelines for treating tobacco dependence omit e-cigarette and HTP dependence, whose users, in any age group, may or may not be motivated to quit. We thus provide recommendations for incorporating the treatment of e-cigarette and HTP use dependence within guidelines for treating tobacco dependence and supporting smoking cessation.

The following aspects should be considered during the development of recommendations for treating e-cigarette and HTP dependence, in parallel to treating tobacco dependence guidelines. Minimal intervention or brief advice for cessation and the 5As strategy should also be tailored for e-cigarette and/or HTP users ${ }^{1,7,8}$. Thus, we recommend:

1. ASK and document all tobacco use, including novel nicotine delivery products (e-cigarettes and HTPs) current/former/never usage. Furthermore, exclusive e-cigarette/HTP or dual use (combustible tobacco product and e-cigarette and/or HTP use concomitantly) frequency and nicotine concentration of the e-liquid would also be valuable to record. Assessment of nicotine/cigarette dependence is routinely done in the clinical diagnosis of tobacco dependence. Several aspects of dependence overlap between nicotine containing e-cigarette and tobacco product use, however, beyond the presence of nicotine in the e-liquid, unique features of e-cigarette use such as flavorings and the device type may also contribute to dependence. Therefore, development of a short and psychometrically appropriate e-cigarette dependence measure for routine clinical assessment is needed.

2. ADVISE e-cigarette and/or HTP users to quit in a clear and personalized manner. Emphasize that novel tobacco product use is addictive, dual use should be avoided, and advise to quit completely.

3. ASSES readiness to quit e-cigarette and/or HTP use. For e-cigarette and/ or HTP users who are not motivated to quit, adaptation of brief motivational interviewing by using the 5Rs (Relevance, Risks, Rewards, Roadblocks, Repetition) strategy in smoking cessation counseling practice may also
AFFILIATION

1 Department of Public Health, Faculty of Medicine, Semmelweis University, Budapest, Hungary 2 National Centre on Addiction and Doping, Istituto Superiore di Sanità (ISS), Rome, Italy

3 Regional Ministry of Health and Families of Andalusia, Directorate General of Public Health and Pharmaceutical Management, Seville, Spain

4 Health Services Management Training Centre, Faculty of Health and Public Administration,

Semmelweis University, Budapest, Hungary

5 George D. Behrakis Research Lab, Hellenic Cancer Society, Athens, Greece

6 Institute of Public Health, The American College of Greece, Athens, Greece

7 Athens Medical Center, Athens, Greece

CORRESPONDING AUTHOR Melinda Pénzes. Department of Public Health, Faculty of Medicine, Semmelweis University, Üllői út 26, H-1085, Budapest, Hungary. E-mail: penzes.melinda@med.semmelweisuniv.hu ORCID: https://orcid.org/00000001-7396-4028

\section{KEYWORDS}

e-cigarette, heated tobacco product, dependence, cessation, guidance

Received: 7 October 2021

Accepted: 8 October 2021 
enhance motivation to quit. For e-cigarette and/or HTP users who are motivated to quit, health professionals should provide assistance in cessation. Studies on the effectiveness of motivational techniques enhancing e-cigarette and/or HTP users' motivation to quit are needed.

4. ASSIST in cessation. For e-cigarette and/or HTP users who are ready to quit, health professionals should provide behavioral counseling and first-line cessation medications. Studies are needed to assess the effectiveness of behavioral counseling and firstline smoking cessation medications in cessation of novel products ${ }^{9}$. If a health professional is not a trained cessation counselor, the patient willing to quit should be referred to a smoking cessation/ quitline service. Novel cessation techniques like online cessation services and smartphone applications may also support cessation, but these need to be rigorously examined.

5. ARRANGE follow-up support. Monitoring the cessation process, adherence to behavioral and/ or pharmacotherapy and recording e-cigarette/ tobacco use status at each follow-up would also be useful. In-person, by phone and/or using novel techniques (online support, mobile apps) at followup could be appropriate, although these should be tested.

Finally, since the science of smoking cessation is still an evolving field, minor and comprehensive updates of cessation guidelines are regularly needed that should be based on the latest scientific evidence and best practices. National circumstances and priorities should be taken into account during the updates of national cessation strategies.

\section{REFERENCES}

1. European Network for Smoking and Tobacco Prevention. Guidelines for treating tobacco dependence. 2020. Accessed February 11, 2021. http://ensp.network/2020guidelines-english-edition/

2. Tobacco Use and Dependence Guideline Panel. Treating tobacco use and dependence: 2008 Update. Clinical Practice Guideline. US Department of Health and Human Services; 2008. Accessed September 21, 2021. https:// www.ncbi.nlm.nih.gov/books/NBK63952/\#!po=5.00000

3. Kapan A, Stefanac S, Sandner I, Haider S, Grabovac I, Dorner TE. Use of electronic cigarettes in European populations: A narrative review. Int J Environ Res Public
Health. 2020;17(6):1971. doi:10.3390/ijerph17061971

4. Ratajczak A, Jankowski P, Strus P, Feleszko W. Heat not burn tobacco product-a new global trend: Impact of heatnot-burn tobacco products on public health, a systematic review. Int J Environ Res Public Health. 2020;17(2):409. doi:10.3390/ijerph17020409

5. Erku DA, Gartner CE, Morphett K, Steadman KJ. Beliefs and self-reported practices of health care professionals regarding electronic nicotine delivery systems: A mixedmethods systematic review and synthesis. Nicotine Tob Res. 2020;22(5):619-629. doi:10.1093/ntr/ntz046

6. Sutanto E, Miller CR, Smith DM, et al. Perceived relative harm of heated tobacco products (IQOS), e-cigarettes, and cigarettes among adults in Canada: Findings from the ITC Project. Tob Induc Dis. 2020;18(September):81. doi:10.18332/tid/127233

7. Prochnow JA. E-cigarettes: A practical, evidence-based guide for advanced practice nurses. J Nurse Pract. 2017;13(7):449-455. doi:10.1016/j.nurpra.2017.03.015

8. Mendelsohn CP. Electronic cigarettes in physician practice: E-cigarettes for tobacco harm reduction. Intern Med J. 2018;48(4):391-396. doi:10.1111/imj.13761

9. Guo FR, Huang HL, Jan CF, Chiang CH, Tsai JS. Combine pharmacotherapy and behavior counseling to quit a heated tobacco product: A case report. Tob Induc Dis. 2021;19(Suppl 1):A207. doi:10.18332/tid/141416

CONFLICTS OF INTEREST

The authors have each completed and submitted an ICMJE Form for Disclosure of Potential Conflicts of Interest. The authors declare that they have no competing interests, financial or otherwise, related to the current work. M. Pénzes and R. Solimini report funding from the project Joint Action 761297/JATC, which has received funding from the European Union's Health Program (2014-2020), paid to her institution.

\section{FUNDING}

This manuscript is part of the project Joint Action 761297/JATC, which has received funding from the European Union's Health Program (2014-2020). The content of this article represents the views of the authors only and it is their responsibility; it does not necessarily reflect the views of the European Commission, the Consumers, Health, Agriculture and Food Executive Agency or any other body of the European Union. The European Commission and the Agency do not accept any responsibility for use that may be made of the information it contains.

ETHICAL APPROVAL AND INFORMED CONSENT

Ethical approval and informed consent were not required for this study.

DATA AVAILABILITY

Data sharing is not applicable to this article as no new data were created.

PROVENANCE AND PEER REVIEW

Commissioned; internally peer reviewed. 